\title{
A MULTI-MODEL APPROACH TO SIMULATE THE STORM SURGE AT PUERTO RICO DUE TO HURRICANES IRMA AND MARIA
}

\author{
David M. Kelly, W.F. Baird \& Associates, dkelly@Baird.com \\ Ewelina Luczko, W.F. Baird \& Associates, eluczko@Baird.com \\ Michael Fullarton, W.F. Baird \& Associates, m fullarton@Baird.com \\ Yahia Kala , W.F. Baird \& Associates, ykala@Baird.com
}

\begin{abstract}
In this paper we present the results of a multimodel approach to simulating the recent storm surges due to hurricanes Irma and Maria. The study focuses on Puerto Rico which, as a consequence of hurricane Maria, experienced storm surge around the entire perimeter of the island. In this study the storm tide is modeled using a variety of state-of-the-art $2 \mathrm{DH}$ numerical models. All models are based on the long wave assumption and employ the Non-Linear Shallow Water (NLSW) equations. The models vary according to the form of the governing NLSW equations that they employ. Differences include linearization and primitive variable or conserved variable (divergence) form. The numerical solution techniques used to solve the governing equations, as well as the options available for the wind, pressure, tidal forcing terms and wetting/drying techniques also vary between (and within) the models.
\end{abstract}

\section{INTRODUCTION}

Coastal flooding due to hurricanes in the US and Caribbean can be incredibly costly with respect to both public safety (including the loss of life) and property damage. Such damage can be of the order of tens of billions of dollars for large hurricane events. Accurate and reliable predictions of surge and flooding in coastal communities, using state-of-the-art numerical models, are thus an essential tool in helping insurers to better understand and quantify the potential risk from hurricanes. Numerical models are used to provide forecasts and hindcasts of storm surge events. Most models are 2D (horizontal) and developed within the framework of the shallow water theory. Although 3D models typically provide a better representation of currents, 2D models are faster to run thus allowing for higher resolution.

In 2017 the island of Puerto Rico was particularly badly affected by hurricanes Irma and Maria. Maria in particular had a devastating effect on the island and was the most intense hurricane to hit Puerto Rico since 1928. Storm surge between $0.5 \mathrm{~m}$ and $1.5 \mathrm{~m}$ occurred around the entire perimeter of the island. This paper compares storm tide predictions for the Island of
Puerto Rico for both hurricane events using several distinct state-of-the-art numerical models

\section{METHODOLOGY}

For initial testing of all of the chosen models a coarse scoping grid comprising under 100,000 nodes was employed. This was then upgraded to a mesh based on the NOAA-NHC HSJ5 PRV6 basin which comprises approx. 450,000 computational nodes. For the curvilinear models, FIST and SLOSH, the NOAA basin is used as is; for the remaining models the existing curvilinear point set was Delaunay triangulated. A number of hurricane storm surge models have been utilized, including the open source models SLOSH, ADCIRC and a TELEMAC-based model (TELEMAC-SS), developed in-house at Baird (which is planned to be made open source and release through the openTELEMAC website), as well as the commercial model MIKE 21 and Baird's own, in-house, Fully Implicit Storm Tide (FIST) Model. The models applied range from finite difference, running on curvilinear grids, to finite volume models running on unstructured triangular meshes. Treatment of the overland flooding ranges from heuristic parameterization of wetting and drying to finite volume models that treat the wetting drying front correctly (within the context of the NLSW equations) as an inter-cell Riemann problem. The model set-up includes the effect of land cover on surge by utilizing spatially varying Manning coefficients based on the USGS national land cover data set. The surge models selected have a variety of parametric approaches to simulate the hurricane wind forcing. In the study presented the Holland (1980) and Myers \& Malkin (1961) models have been utilized. Moreover, several different approaches for parameterizing the wind stress, such as the well known Garratt (1977) and Large $\&$ Pond (1981) approaches, are explored in the open source and Baird in-house models. The effect of short waves, incorporated via a spectral wave model, on the surge is also investigated. All of the storm tide models are run on the same computational domain. An overview of the salient features of each model for both the surge and wind forcing will be presented. 


\section{RESULTS}

Figure 1 shows an illustrative time-series of the free surface for the surge due to hurricane Irma. Both the NOAA gauge data and the predicted free surface time-series, obtained using the newly developed TELEMAC-SS, are shown. Results shown were obtained by running the TELEMAC-SS model in forecast mode (i.e. essentially uncalibrated) without the effect of short wave forcing (typically obtained through the use of a spectral wave model). Figure 2 gives illustrative results for the maximum surge due to hurricane Maria as predicted by the TELEMAC-SS model for the island of Puerto Rico. For the results presented in Figure 2 the model was run on the NOAA HSJ5 basin in forecast mode with tides but without the contribution due to short waves.

At the conference model predictions for the various models with a number of different combinations inputs, and parameterizations of inputs, will be presented. Presented results will take the form of time series comparisons with NOAA gauge data and inundation extents.
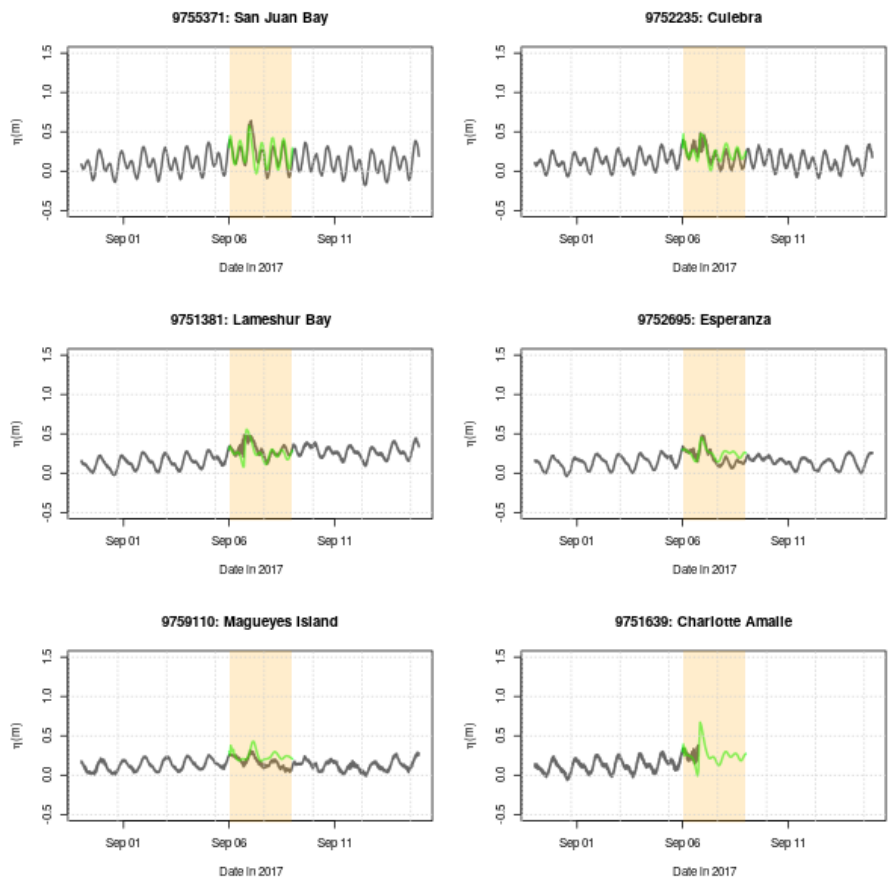

Figure 1 - Time Series for Puerto Rico obtained using the newly developed TELEMAC-SS model in forecast mode (without short waves) on the NOAA HSJ 5 basin.

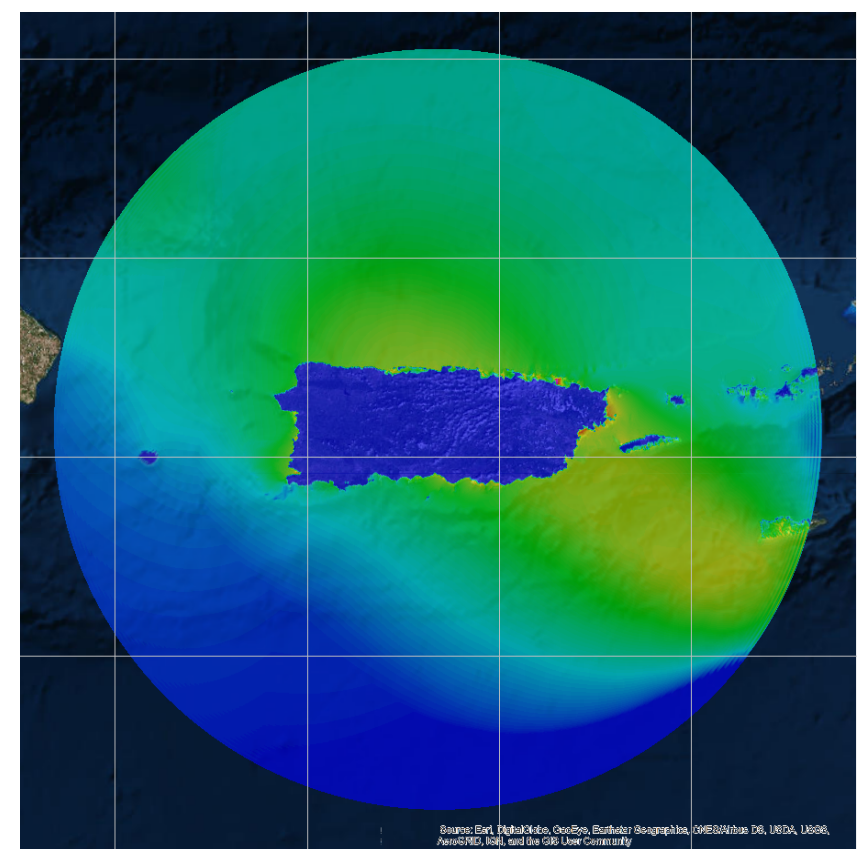

Figure 2 - Maximum Envelope of Observed Water (MEOW) due to hurricane Maria and tide around Puerto Rico obtained using the TELEMAC-SS model on the NOAA HSJ 5 basin ( 0 to $2 \mathrm{~m}$ blue to red).

\section{CONCLUSIONS}

At the conference conclusions relating to the computational cost, robustness of the models (including numerical stability) as well as the predictive skill. The practicality of the chosen models for storm surge modeling will also be be discussed.

\section{REFERENCES}

Garratt (1977) Review of Drag Coefficients over Oceans and Continents Monthly Weather Review, 105, pp. 915-929.

Large and Pond (1981) Open Ocean Momentum Flux Measurements in Relatively Strong Winds, J. Phys. Oceanogr. 11, 464-482.

Holland (1980). An Analytic Model of the Wind and Pressure Profiles in Hurricanes. Monthly Weather Review, 108, pp. 1212-1218.

Myers and Malkin (1961) Some Properties of Hurricane Wind Fields as Deduced from Trajectories. Washington DC: NOAA, 43p. 